\title{
Real-rooted polynomials via generalized Bell umbra
}

\author{
Abdelkader Benyattou and Miloud Mihoubi \\ Faculty of Mathematics, USTHB, RECITS Laboratory \\ P. O. 32 Box 32, El Alia 16111, Algiers, Algeria \\ e-mails: abdelkaderbenyattou@gmail.com, \\ mmihoubi@usthb.dz
}

Received: 7 September 2018

Accepted: 19 March 2019

\begin{abstract}
In this paper, by the generalized Bell umbra and Rolle's theorem, we give some results on the real rootedness of polynomials. Some applications on partition polynomials are considered. Our results are illustrated by some comprehensive examples.
\end{abstract}

Keywords: Polynomials with real zeros, Generalized Bell umbra, Partition polynomials. 2010 Mathematics Subject Classification: 11B73, 30C15.

\section{Introduction}

The real rootedness of polynomials has attracted researchers great interest. One of the reasons is that any polynomial of real zeros implies the log-concavity and the unimodality of its coefficients, which appear in various fields of mathematics, see [6, 18]. In this paper, we investigate the properties of the generalized Bell umbra and Rolle's theorem to give a result on the polynomials of real zeros. Partial and auxiliary results on the $\sigma$-polynomials of graphs and on a class of polynomials linked to the partial $r$-Bell polynomials are considered.

To use later, recall that the $n$-th Bell polynomial $\mathcal{B}_{n}(x)$ and the $n$-th $r$-Bell polynomial $\mathcal{B}_{n, r}(x)$ can be defined by Dobinski's formula

$$
\mathcal{B}_{n}(x)=e^{-x} \sum_{j \geq 0} j^{n} \frac{x^{j}}{j !}, \quad \mathcal{B}_{n, r}(x)=e^{-x} \sum_{j \geq 0}(j+r)^{n} \frac{x^{j}}{j !}
$$


and $\mathbf{B}_{\mathbf{x}}^{n}=\mathcal{B}_{n}(x)$ be the generalized Bell umbra introduced by Sun et al. [19]. It is known that, for any polynomial $f$ and any integer $n \geq 0$, the generalized Bell umbra satisfies [19]

$$
\left(\mathbf{B}_{\mathbf{x}}\right)_{n} f\left(\mathbf{B}_{\mathbf{x}}\right)=x^{n} f\left(\mathbf{B}_{\mathbf{x}}+n\right) \text {, }
$$

where $(x)_{n}=x(x-1) \cdots(x-n+1)$ if $n \geq 1$ and $(x)_{0}=1$. In particular, we have

$$
\mathbf{B}_{\mathbf{x}}^{n+1}=x\left(\mathbf{B}_{\mathbf{x}}+1\right)^{n} \text { and }\left(\mathbf{B}_{\mathbf{x}}\right)_{n}=x^{n} .
$$

Some polynomials have closed forms in terms of $\mathbf{B}_{\mathbf{x}}$ such the polynomials [4]

$$
\mathcal{B}_{n, r}(x)=\left(\mathbf{B}_{\mathbf{x}}+r\right)^{n}=x^{-r}\left(\mathbf{B}_{\mathbf{x}}\right)_{r} \mathbf{B}_{\mathbf{x}}^{n}, \quad \mathcal{L}_{n}(x)=\left(\mathbf{B}_{\mathbf{x}}+n-1\right)_{n},
$$

and for $r_{1} \leq \cdots \leq r_{p}$ be non-negative integers, we also have [4, 12]:

$$
\mathcal{B}_{n ; r_{1}, \ldots, r_{p}}(x)=\left(\mathbf{B}_{\mathbf{x}}+r_{p}\right)_{r_{p}} \cdots\left(\mathbf{B}_{\mathbf{x}}+r_{p}\right)_{r_{1}}\left(\mathbf{B}_{\mathbf{x}}+r_{p}\right)^{n}=x^{-r_{p}}\left(\mathbf{B}_{\mathbf{x}}\right)_{r_{p}} \cdots\left(\mathbf{B}_{\mathbf{x}}\right)_{r_{1}} \mathbf{B}_{\mathbf{x}}^{n},
$$

where $\mathcal{B}_{n ; r_{1}, \ldots, r_{p}}(x)$ are the $\left(r_{1}, \ldots, r_{p}\right)$-Bell polynomials [12] and $\mathcal{L}_{n}(x)$ are the Lah polynomials. More generally, for any polynomial $f$, there holds

$$
e^{-x} \sum_{k \geq 0} f(k) \frac{x^{k}}{k !}=f\left(\mathbf{B}_{\mathbf{x}}\right) .
$$

For further information about umbral calculus on Bell polynomials, one can also see [4, 9, 10]. The paper is organized as follows. In the next section we give a result on polynomials with real zeros. In the last section we present applications on a class of polynomials linked to the partial $r$-Bell polynomials [16].

\section{Real-rooted polynomials via generalized Bell umbra}

Let $\mathrm{RZ}$ be the set of the real polynomials having only real zeros.

The principal main result of this paper is given by the following theorem.

Theorem 1. Let $f$ be a polynomial with real coefficients such that $f\left(\mathbf{B}_{\mathbf{x}}\right) \in \mathbf{R Z}$. Then, for any non-negative integer $r$, there holds

$$
\left(\mathbf{B}_{\mathbf{x}}\right)_{r} f\left(\mathbf{B}_{\mathbf{x}}\right) \in \mathbf{R Z}
$$

Furthermore, for any non-negative integers $r_{1}, \ldots, r_{p}$, there holds

$$
\left(\mathbf{B}_{\mathbf{x}}\right)_{r_{p}} \cdots\left(\mathbf{B}_{\mathbf{x}}\right)_{r_{1}} f\left(\mathbf{B}_{\mathbf{x}}\right) \in \mathbf{R Z} .
$$

Proof. From the identity $f\left(\mathbf{B}_{\mathbf{x}}\right)=e^{-x} \sum_{k \geq 0} f(k) \frac{x^{k}}{k !}[4]$ it follows

$$
\frac{d}{d x}\left(e^{x} f\left(\mathbf{B}_{\mathbf{x}}+r-1\right)\right)=\frac{d}{d x}\left(\sum_{k \geq 0} f(k+r-1) \frac{x^{k}}{k !}\right)=\sum_{k \geq 0} f(k+r) \frac{x^{k}}{k !}=e^{x} f\left(\mathbf{B}_{\mathbf{x}}+r\right) .
$$

The proof can be obtained by induction on $r$ and by application of Rolle's theorem on the function $e^{x} f\left(\mathbf{B}_{\mathbf{x}}+r-1\right)$. More generally, since $g(x):=\left(\mathbf{B}_{\mathbf{x}}\right)_{r_{1}} f\left(\mathbf{B}_{\mathbf{x}}\right) \in \mathbf{R Z}$, then the polynomial $\left(\mathbf{B}_{\mathbf{x}}\right)_{r_{2}} g\left(\mathbf{B}_{\mathbf{x}}\right)=\left(\mathbf{B}_{\mathbf{x}}\right)_{r_{2}}\left(\mathbf{B}_{\mathbf{x}}\right)_{r_{1}} f\left(\mathbf{B}_{\mathbf{x}}\right)$ has only real zeros, and so on. 
Example 1. For $f(x)=x^{n}$ we get $f\left(\mathbf{B}_{\mathbf{x}}\right)=\mathcal{B}_{n}(x) \in \mathbf{R Z}$. We deduce from Theorem 1 the known result [11, Th. 6]

$$
\left(\mathbf{B}_{\mathbf{x}}\right)_{r} \mathbf{B}_{\mathbf{x}}^{n}=x^{r} \mathcal{B}_{n ; r}(x) \in \mathbf{R Z}
$$

and the known result [12] (see also [4, 15])

$$
\left(\mathbf{B}_{\mathbf{x}}\right)_{r_{p}} \cdots\left(\mathbf{B}_{\mathbf{x}}\right)_{r_{1}} \mathbf{B}_{\mathbf{x}}^{n}=x^{\max \left\{r_{1}, \ldots, r_{p}\right\}} \mathcal{B}_{n ; r_{1}, \ldots, r_{p}}(x) \in \mathbf{R Z} .
$$

Example 2. For $f(x)=(x)_{n}$ we get $f\left(\mathbf{B}_{\mathbf{x}}\right)=x^{n} \in \mathbf{R Z}$. It follows from Theorem 1 that the polynomial

$$
\left(\mathbf{B}_{\mathbf{x}}\right)_{r} f\left(\mathbf{B}_{\mathbf{x}}\right)=x^{r}\left(\mathbf{B}_{\mathbf{x}}+r\right)_{n}=x^{r} \sum_{k=0}^{\min (n, r)}\left(\begin{array}{l}
n \\
k
\end{array}\right) \frac{r !}{(r-k) !} x^{n-k}
$$

is in RZ. More generally, the polynomial

$$
\left(\mathbf{B}_{\mathbf{x}}\right)_{r_{p}} \cdots\left(\mathbf{B}_{\mathbf{x}}\right)_{r_{1}}\left(\mathbf{B}_{\mathbf{x}}\right)_{n}=x^{\max \left\{r_{1}, \ldots, r_{p}, n\right\}} \mathcal{B}_{0 ; r_{1}, \ldots, r_{p}, n}(x)
$$

is in RZ.

Example 3. For $f(x)=(x+n-1)_{n}$ we get $f\left(\mathbf{B}_{\mathbf{x}}\right)=\left(\mathbf{B}_{\mathbf{x}}+n-1\right)_{n}=\mathcal{L}_{n}(x) \in \mathbf{R Z}$ [1]. It follows from Theorem 1 that the polynomial

$$
\begin{aligned}
\left(\mathbf{B}_{\mathbf{x}}\right)_{r_{p}} \cdots\left(\mathbf{B}_{\mathbf{x}}\right)_{r_{1}}\left(\mathbf{B}_{\mathbf{x}}+n-1\right)_{n} & =\sum_{k=0}^{n}\left(\begin{array}{l}
n \\
k
\end{array}\right)(n-1)_{n-k}\left(\mathbf{B}_{\mathbf{x}}\right)_{r_{p}} \cdots\left(\mathbf{B}_{\mathbf{x}}\right)_{r_{1}}\left(\mathbf{B}_{\mathbf{x}}\right)_{k} \\
& =\sum_{k=0}^{n} L(n, k) x^{\max \left\{k, r_{1}, \ldots, r_{p}\right\}} \mathcal{B}_{0 ; k, r_{1}, \ldots, r_{p}}(x)
\end{aligned}
$$

is in $\mathbf{R Z}$, where $L(n, k)$ are the Lah numbers.

To give an application on the $\sigma$-polynomial associated to a graph, let $\lambda$ be a positive integer and recall that a $\lambda$-coloring of a simple graph $G$ is a mapping $f: V \rightarrow\{1,2, \ldots, \lambda\}$ where $f(u) \neq f(v)$ whenever the vertices $u$ and $v$ are adjacent in $G$. Two $\lambda$-colorings $f$ and $g$ of $G$ are distinct if $f(x) \neq g(x)$ for some vertex $x$ in $G$, and, the number of $\lambda$-colorings of $G$ is called the chromatic polynomial $P(G, \lambda)$. The chromatic polynomial can be defined as

$$
f(\lambda)=\sum_{k=0}^{n} \alpha_{k}(G)(\lambda)_{k},
$$

where $\alpha_{k}(G)$ is the number of ways of partitioning $V$ into $k$ nonempty sets. The $\sigma$-polynomial associated to $G$ is

$$
\sum_{k=0}^{n} \alpha_{k}(G) x^{k}=\sum_{k=0}^{n} \alpha_{k}(G)\left(\mathbf{B}_{\mathbf{x}}\right)_{k}=f\left(\mathbf{B}_{\mathbf{x}}\right) .
$$

For more information about chromatic polynomials, see [8].

Corollary 2. If the $\sigma$-polynomial $f\left(\mathbf{B}_{\mathbf{x}}\right)$ of a graph $G$ is in $\mathbf{R Z}$, then the $\sigma$-polynomial

$$
\left(\mathbf{B}_{\mathbf{x}}\right)_{r_{1}} \cdots\left(\mathbf{B}_{\mathbf{x}}\right)_{r_{p}} f\left(\mathbf{B}_{\mathbf{x}}\right)
$$

of the graph $G \cup K_{r_{1}} \cup \cdots \cup K_{r_{p}}$ is in $\mathbf{R Z}$, where $K_{r}$ is the complete graph of $r$ vertices. 
Example 4. A tree $T_{n}$ of $n(\geq 1)$ vertices has chromatic polynomial $f(\lambda)=\lambda(\lambda-1)^{n-1}$. By the identity $\left(\mathbf{B}_{\mathbf{x}}\right)_{n} f\left(\mathbf{B}_{\mathbf{x}}\right)=x^{n} f\left(\mathbf{B}_{\mathbf{x}}+n\right)$, the $\sigma$-polynomial of $T_{n}$ must be

$$
f\left(\mathbf{B}_{\mathbf{x}}\right)=\mathbf{B}_{\mathbf{x}}\left(\mathbf{B}_{\mathbf{x}}-1\right)^{n-1}=x \mathbf{B}_{\mathbf{x}}^{n-1}=x \mathcal{B}_{n-1}(x)
$$

which is in $\mathbf{R Z}$. Then, the $\sigma$-polynomial of the graph $T_{n} \cup K_{r}$ is in $\mathbf{R Z}$, and is to be

$$
\left(\mathbf{B}_{\mathbf{x}}\right)_{r} f\left(\mathbf{B}_{\mathbf{x}}\right)=x^{r}\left(\mathbf{B}_{\mathbf{x}}+r\right)\left(\mathbf{B}_{\mathbf{x}}+r-1\right)^{n-1}=x^{r}\left[x \mathcal{B}_{n-1, r}(x)+r \mathcal{B}_{n-1, r-1}(x)\right] .
$$

\subsection{Polynomials linked to the partial $r$-Bell polynomials}

We present in this section some applications of Theorem 1 on a class of polynomials linked to the partial $r$-Bell polynomials. Indeed, recall that the $(n, k)$-th partial $r$-Bell polynomial

$$
B_{n+r, k+r}^{(r)}(\mathbf{a} ; \mathbf{b}):=B_{n+r, k+r}^{(r)}\left(a_{1}, a_{2}, \ldots ; b_{1}, b_{2}, \ldots\right)
$$

introduced by Mihoubi et al. [16] (see also [17]) can be defined by

$$
\sum_{n \geq k} B_{n+r, k+r}^{(r)}(\mathbf{a} ; \mathbf{b}) \frac{t^{n}}{n !}=\frac{1}{k !}\left(\sum_{j \geq 1} a_{j} \frac{t^{j}}{j !}\right)^{k}\left(\sum_{j \geq 0} b_{j+1} \frac{t^{j}}{j !}\right)^{r}
$$

This polynomial presents an extension of the $(n, k)$-th partial Bell polynomial

$$
B_{n, k}\left(a_{1}, a_{2}, \ldots\right):=B_{n, k}(\mathbf{a})
$$

introduced by Bell [2] and studied later by several authors, see for example [7, 13, 14]).

Let $\left(a_{n}\right)$ and $\left(b_{n}\right)$ be two the sequences of real numbers linked as follows

$$
\varphi(t)=\sum_{n \geq 1} a_{n} \frac{t^{n}}{n !}, \quad 1+\varphi(t)=\sum_{n \geq 0} b_{n+1} \frac{t^{n}}{n !},
$$

Here $\mathbf{b}=\mathbf{e}+L \mathbf{a}$, where $\mathbf{e}=(1,0,0, \ldots), \mathbf{a}=\left(a_{1}, a_{2}, \ldots\right)$, and the sequence $\left(L^{n} \mathbf{a}\right)$ is defined by $L^{0} \mathbf{a}=\left(a_{1}, a_{2}, \ldots\right), L \mathbf{a}=\left(0, a_{1}, a_{2}, \ldots\right), L^{2} \mathbf{a}=\left(0,0, a_{1}, a_{2}, \ldots\right)$ and so on.

Proposition 3. Let $r$ be a non-negative integer and let $\mathcal{V}_{n, r}(x)$ and $\mathcal{V}_{n}(x)$ be the polynomials defined by

$$
\mathcal{V}_{n, r}(x)=\sum_{k=0}^{n} B_{n+r, k+r}^{(r)}(\mathbf{a} ; \mathbf{e}+L \mathbf{a}) x^{k}, \quad \mathcal{V}_{n}(x)=\mathcal{V}_{n, 0}(x)=\sum_{k=0}^{n} B_{n, k}(\mathbf{a}) x^{k}
$$

If $\mathcal{V}_{n}(x) \in \mathbf{R Z}$, then $\mathcal{V}_{n, r}(x) \in \mathbf{R Z}$.

Proof. From [16, Th. 4] we have

$$
\begin{aligned}
\sum_{n \geq 0} B_{n+r, k+r}^{(r)}(\mathbf{a} ; \mathbf{e}+L \mathbf{a}) \frac{t^{n}}{n !} & =\frac{1}{k !}(\varphi(t))^{k}(1+\varphi(t))^{r}, \\
\sum_{n \geq 0} \mathcal{V}_{n, r}(x) \frac{t^{n}}{n !} & =(1+\varphi(t))^{r} \exp (x \varphi(t)) .
\end{aligned}
$$


Then, for $f_{n}(x):=\sum_{k=0}^{n} B_{n, k}(\mathbf{a})(x)_{k}$ we get $f_{n}\left(\mathbf{B}_{\mathbf{x}}+r\right)=\mathcal{V}_{n, r}(x)$. Indeed, we have

$$
\begin{aligned}
\sum_{n \geq 0} f_{n}\left(\mathbf{B}_{\mathbf{x}}+r\right) \frac{t^{n}}{n !} & =\sum_{k \geq 0}\left(\mathbf{B}_{\mathbf{x}}+r\right)_{k} \sum_{n \geq k} B_{n, k}(\mathbf{a}) \frac{t^{n}}{n !} \\
& =\sum_{k \geq 0}\left(\begin{array}{c}
\mathbf{B}_{\mathbf{x}}+r \\
k
\end{array}\right)(\varphi(t))^{k} \\
& =(1+\varphi(t))^{\mathbf{B}_{\mathbf{x}}+r} \\
& =(1+\varphi(t))^{r} \sum_{n \geq 0} f_{n}\left(\mathbf{B}_{\mathbf{x}}\right) \frac{t^{n}}{n !} \\
& =(1+\varphi(t))^{r} \exp (x \varphi(t))
\end{aligned}
$$

which is the exponential generating function of the sequence $\left(\mathcal{V}_{n, r}(x)\right)$.

Hence, the application of Theorem 1 completes the proof.

Corollary 4. For $\mathbf{a}=(1,1,1, \ldots)$, the following polynomials are in $\mathbf{R Z}$

$$
\mathcal{V}_{n, r}(x)=\sum_{k=0}^{n} B_{n+r, k+r}^{(r)}\left(L \mathbf{a} ; \mathbf{e}+L^{2} \mathbf{a}\right) x^{k}, \quad \mathcal{U}_{n, r}(x)=\sum_{k=0}^{n} B_{n+r, k+r}^{(r)}\left(L^{2} \mathbf{a} ; \mathbf{e}+L^{3} \mathbf{a}\right) x^{k}
$$

Proof. The 2-associated and 3-associated Bell polynomials

$$
\mathcal{V}_{n}(x)=\sum_{k=0}^{n}\left\{\begin{array}{l}
n \\
k
\end{array}\right\}^{(2)} x^{k}=\sum_{k=0}^{n} B_{n, k}(L \mathbf{a}) x^{k}, \quad \mathcal{U}_{n}(x)=\sum_{k=0}^{n}\left\{\begin{array}{l}
n \\
k
\end{array}\right\}^{(3)} x^{k}=\sum_{k=0}^{n} B_{n, k}\left(L^{2} \mathbf{a}\right) x^{k}
$$

are in $\mathbf{R Z}$, where

$$
\sum_{n \geq k}\left\{\begin{array}{l}
n \\
k
\end{array}\right\}^{(2)} \frac{t^{n}}{n !}=\frac{1}{k !}\left(e^{t}-1-t\right)^{k}, \quad \sum_{n \geq k}\left\{\begin{array}{l}
n \\
k
\end{array}\right\}^{(3)} \frac{t^{n}}{n !}=\frac{1}{k !}\left(e^{t}-1-t-\frac{t^{2}}{2}\right)^{k}
$$

see $[5,20]$. So, the corollary follows from Proposition 3.

The potential polynomials used by Bender and Canfield [3, Th. 1] to study the log-concavity and log-convexity of their coefficients are used here to define sequences of polynomials by the generalized Bell umbra for which we deduce an easy application of Theorem 1.

Lemma 5. Let $\left(A_{n}(x)\right)$ be a sequence of polynomials defined by

$$
1+\sum_{n \geq 1} A_{n}(x) \frac{t^{n}}{n !}=\exp (x h(t)), \quad h(t)=\sum_{j \geq 1} a_{j} \frac{t^{j}}{j !}
$$

and let $\left(A_{n}^{(s)}(x)\right)$ be a sequence of polynomials defined by

$$
A_{n}^{(0)}(x)=A_{n}(x), A_{n}^{(s)}(x)=A_{n}^{(s-1)}\left(\mathbf{B}_{\mathbf{x}}\right), \quad s \geq 1 .
$$

Then

$$
1+\sum_{n \geq 1} A_{n}^{(s)}(x) \frac{t^{n}}{n !}=\exp \left(x \sum_{j \geq 1} A_{j}^{(s-1)}(1) \frac{t^{j}}{j !}\right), s \geq 1 .
$$


Proof. We proceed by induction on $s \geq 1$. Since

$$
\begin{aligned}
\sum_{n \geq 0} A_{n}^{(1)}(x) \frac{t^{n}}{n !} & =\sum_{n \geq 0} A_{n}^{(0)}\left(\mathbf{B}_{\mathbf{x}}\right) \frac{t^{n}}{n !} \\
& =\exp \left(\mathbf{B}_{\mathbf{x}} h(t)\right) \\
& =\sum_{n \geq 0} \frac{\mathcal{B}_{n}(x)}{n !}(h(t))^{n} \\
& =\exp (x(\exp (h(t))-1)) \\
& =\exp \left(x \sum_{n \geq 1} A_{n}^{(0)}(1) \frac{t^{n}}{n !}\right)
\end{aligned}
$$

it follows that the desired identity is true for $s=1$. Assume it is true for $s, s \geq 1$. Then

$$
\begin{aligned}
\sum_{n \geq 0} A_{n}^{(s+1)}(x) \frac{t^{n}}{n !} & =\sum_{n \geq 0} A_{n}^{(s)}\left(\mathbf{B}_{\mathbf{x}}\right) \frac{t^{n}}{n !} \\
& =\exp \left(\mathbf{B}_{\mathbf{x}} \sum_{j \geq 1} A_{j}^{(s-1)}(1) \frac{t^{j}}{j !}\right) \\
& =\sum_{n \geq 0} \frac{\mathcal{B}_{n}(x)}{n !}\left(\sum_{j \geq 1} A_{j}^{(s-1)}(1) \frac{t^{j}}{j !}\right)^{n} \\
& =\exp \left(x\left(\exp \left(\sum_{j \geq 1} A_{j}^{(s-1)}(1) \frac{t^{j}}{j !}\right)-1\right)\right) \\
& =\exp \left(x \sum_{n \geq 1} A_{n}^{(s)}(1) \frac{t^{n}}{n !}\right)
\end{aligned}
$$

which proves the induction step.

Lemma 5 and Proposition 3 state that we have:

Corollary 6. Let $r, s$ be non-negative integers, $\mathbf{a}_{s}=\left(A_{1}^{(s)}(1), A_{2}^{(s)}(1), \ldots\right)$ and let $\mathcal{V}_{n, r}^{(s)}(x)$ and $\mathcal{V}_{n}^{(s)}(x)$ be the polynomials defined by

$$
\mathcal{V}_{n, r}^{(s)}(x)=\sum_{k=0}^{n} B_{n+r, k+r}^{(r)}\left(\mathbf{a}_{s} ; \mathbf{e}+L \mathbf{a}_{s}\right) x^{k}, \quad \mathcal{V}_{n}^{(s)}(x)=\mathcal{V}_{n, 0}^{(s)}(x)=\sum_{k=0}^{n} B_{n, k}\left(\mathbf{a}_{s}\right) x^{k}
$$

If $\mathcal{V}_{n}^{(s)}(x) \in \mathbf{R Z}$, then $\mathcal{V}_{n, r}^{(s)}(x) \in \mathbf{R Z}$

Example 5. For $a_{n}=(n-1) !, n \geq 1$, we get $h(t)=-\ln (1-t)$. Then, under the notations of Lemma 5 and Corollary 6, we obtain

$$
\begin{aligned}
A_{n}^{(0)}(x) & =x(x+1) \cdots(x+n-1) \\
\mathbf{a}_{0} & =\left(A_{1}^{(0)}(1), A_{2}^{(0)}(1), \ldots\right)=(1 !, 2 !, \ldots)
\end{aligned}
$$


and

$$
\mathcal{V}_{n, 0}^{(0)}(x)=\sum_{k=0}^{n} B_{n, k}\left(\mathbf{a}_{0}\right) x^{k}=\sum_{k=0}^{n} L(n, k) x^{k}=\mathcal{L}_{n}(x)
$$

which is in $\mathbf{R Z}$, then the polynomial

$$
\mathcal{V}_{n, r}^{(0)}(x)=\sum_{k=0}^{n} B_{n+r, k+r}^{(r)}\left(\mathbf{a}_{0} ; \mathbf{e}+L \mathbf{a}_{0}\right) x^{k}=\sum_{k=0}^{n} \frac{n !}{k !}\left(\begin{array}{c}
n+r-1 \\
k+r-1
\end{array}\right) x^{k}, \quad r \geq 1,
$$

is also in $\mathbf{R Z}$.

A further result on exponential polynomials with real roots is given by the following proposition.

Proposition 7. Let $r$ be a non-negative integer and $\left(f_{n}^{(r)}(x)\right)$ be the sequence defined by

$$
\sum_{n \geq 0} f_{n}^{(r)}(x) \frac{t^{n}}{n !}=F(t)(h(t))^{r} \exp (x h(t)), \quad h(t)=\sum_{j \geq 1} a_{j} \frac{t^{j}}{j !},
$$

for some power series $F$. Then, for $r \leq n-1$, if the polynomial $f_{n}^{(0)}(x)$ is of degree $n$ and is in $\mathrm{RZ}$, then

$$
f_{n}^{(r)}(x)=r ! \sum_{k=r}^{n}\left(\begin{array}{l}
n \\
k
\end{array}\right) B_{k, r}(\mathbf{a}) f_{n-k}^{(0)}(x) \in \mathbf{R Z} .
$$

Proof. By definition of the sequence $\left(f_{n}^{(r)}(x)\right)$ it follows that $f_{n}^{(r)}(x)=\frac{d}{d x} f_{n}^{(r-1)}(x)$. This shows that the polynomial $f_{n}^{(r)}(x)$ is of degree $n-r$. The proof can be deduced by induction on $r$ and by application of Rolle's theorem.

Example 6. Let $\left(f_{n}^{(r)}(x)\right)$ be the sequence defined by

$$
\sum_{n \geq 0} f_{n}^{(r)}(x) \frac{t^{n}}{n !}=\left(e^{t}-1\right)^{r} \exp \left(x\left(e^{t}-1\right)\right) .
$$

Then, the polynomial $f_{n}^{(r)}(x)=r ! \sum_{k=r}^{n}\left(\begin{array}{l}n \\ k\end{array}\right)\left\{\begin{array}{l}k \\ r\end{array}\right\} \mathcal{B}_{n-k}(x)$ is in RZ, where $\left\{\begin{array}{l}k \\ r\end{array}\right\}$ are the Stirling numbers of the second kind.

Example 7. Let $\left(f_{n}^{(r)}(x)\right)$ be the sequence defined by

$$
\sum_{n \geq 0} f_{n}^{(r)}(x) \frac{t^{n}}{n !}=(\ln (1+t))^{r} \exp (x(\ln (1+t))) .
$$

Then, the polynomial $f_{n}^{(r)}(x)=r ! \sum_{k=r}^{n}(-1)^{k-r}\left(\begin{array}{l}n \\ k\end{array}\right)\left[\begin{array}{l}k \\ r\end{array}\right](x)_{n-k}$ is in RZ.

Example 8. Let $\left(f_{n}^{(r)}(x)\right)$ be the sequence defined by

$$
\sum_{n \geq 0} f_{n}^{(r)}(x) \frac{t^{n}}{n !}=\frac{1}{r !}\left(\frac{t}{1-t}\right)^{r} \exp \left(\frac{x t}{1-t}\right) .
$$

Then, the polynomial $f_{n}^{(r)}(x)=r ! \sum_{k=r}^{n}(-1)^{k-r}\left(\begin{array}{l}n \\ k\end{array}\right) L(k, r) \mathcal{L}_{n-k}(x)$ is in RZ. 


\section{References}

[1] Ahuja, J. C. \& Enneking, E. A. (1979). Concavity property and a recurrence relation for associated Lah numbers, The Fibonacci Quarterly, 17, 158-161.

[2] Bell, E.T. (1934). Exponential polynomials, Ann. Math., 35, 258-277.

[3] Bender, E. A. \& Canfield, E. R. (1996). Log-concanvity and related properties of the cycle index polynomials, J. Combin. Theory Ser. A, 74, 57-70.

[4] Benyattou, A. \& Mihoubi, M. (2018). Curious congruences related to the Bell polynomials, Quaest. Math., 41 (3), 437-448.

[5] Bóna, M., \& Mező, I. (2016). Real zeros and partitions without singleton blocks, European J. Combin., 51, 500-510.

[6] Brenti, F. (1994). Log-concave and unimodal sequences in algebra, combinatorics, and geometry: an update, Contemp. Math., 178, 71-89.

[7] Comtet, L. (1974). Advanced Combinatorics, D. Reidel Publishing Company, DordrechtHolland, Boston-USA, 133-175.

[8] Dong, F. M., Koh, K. M. \& Teo, K. L. (2005). Chromatic polynomials and chromaticity of graphs, World Scientific, British library.

[9] Gertsch, A. \& Robert, A. M. (1996). Some congruences concerning the Bell numbers, Bull. Belg. Math. Soc. Simon Stevin, 3, 467-475.

[10] Gessel, I.M. (2003). Applications of the classical umbral calculus, Algebra Universalis, 49, 397-434.

[11] Mező, I. (2008). On the maximum of $r$-Stirling numbers,Adv. Appl. Math., 41, 293-306.

[12] Maamra, M. S. \& Mihoubi, M. (2014). The $\left(r_{1}, \ldots, r_{p}\right)$-Bell polynomials, Integers, 14, Article A34.

[13] Mihoubi, M. (2008). Bell polynomials and binomial type sequences, Discrete Math., 308, 2450-2459.

[14] Mihoubi, M. (2013). The role of binomial type sequences in determination identities for Bell polynomials, Ars Combin., 111, 323-337.

[15] Mihoubi, M. \& Maamra, M. S. (2012). The $\left(r_{1}, \ldots, r_{p}\right)$-Stirling numbers of the second kind, Integers, 12, Article A35.

[16] Mihoubi, M. \& Rahmani, M. (2017). The partial $r$-Bell polynomials, Afr. Mat., 28 (7-8), 1167-1183. 
[17] Shattuck, M. (2017). Some combinatorial formulas for the partial $r$-Bell polynomials, Notes Number Th. Discr. Math., 23 (1), 63-76.

[18] Stanley, R. P. (1989). Log-concave and unimodal sequences in algebra, combinatorics, and geometry, Ann. New York Acad. Sci., 576, 500-534.

[19] Sun, Y. , Wu, X. \& Zhuang, J. (2013). Congruences on the Bell polynomials and the derangement polynomials, J. Num. Theory, 133, 1564-1571.

[20] Tebtoub, A.F. (2017). Aspects combinatoires liés à la monotonie des suites classiques, Doctorat thesis, USTHB, 2017. 